\title{
The Protective Effect of Heme Oxygenase-1 against Intestinal Barrier Dysfunction in Cholestatic Liver Injury Is Associated with NF-kB Inhibition
}

\author{
Lijing Zhang, ${ }^{1}$ Zhenling Zhang, ${ }^{1}$ Bojia Liu, ${ }^{1}$ Yanling Jin, ${ }^{2}$ Yan Tian, ${ }^{3}$ Yi Xin ${ }^{4}$ and Zhijun Duan ${ }^{1}$
}

${ }^{1}$ Department of Gastroenterology, ${ }^{2}$ Department of Pathology, First Affiliated Hospital of Dalian Medical University, Dalian, China; ${ }^{3}$ College of Pharmacy, and ${ }^{4}$ Department of Biochemistry and Molecular Biology, Dalian Medical University, Dalian, China

\begin{abstract}
Heme oxygenase-1 (HO-1) is reported to protect against liver injury, but little is known about its effect on the intestinal barrier in cholestatic liver injury. In this study, we investigated the effects of $\mathrm{HO}-1$ and its enzymatic by-product on intestinal barrier dysfunction in bile duct ligation (BDL) rats and explored the possible mechanism. The HO-1 inducer cobalt protoporphyrin (CoPP) and carbon monoxide-releasing molecule-2 (CORM-2) were used; the expression levels of tight junction (TJ) proteins, intestinal inflammation and NF-kB p65 were measured. For an in vitro experiment, stable Caco-2 cell lines were constructed, one overexpressed the HO-1 gene and another with that gene knocked down, and the specific NF-kB inhibitor JSH-23 was used. CoPP and CORM-2 treatment alleviated liver and intestinal mucosa injury in BDL rats; improved ZO-1, claudin-1 and PCNA expression; and reduced cell apoptosis and intestinal interleukin-6 (IL-6) expression. In vitro studies confirmed that HO-1, ZO-1 and occludin were overexpressed in $\mathrm{HO}$-1-transfected Caco-2 cells, while decreased in the short hairpin $\mathrm{HO}$ (sh-HO-1) group. JSH-23 significantly increased occludin expression in both the HO-1 overexpression and sh-HO-1 groups, compared with their respective controls. HO-1 overexpression also inhibited the nuclear translocation of NF-kB p65 after lipopolysaccharide (LPS) treatment. Additionally, phospho-p65 expression in sh-HO- 1 cells was significantly increased compared with that of the $\mathrm{HO}-1$ overexpression group. In conclusion, $\mathrm{HO}-1$ and CORM-2 improved intestinal epithelial barrier function in BDL-induced cholestatic liver injury mainly by restoring TJ, reducing cell apoptosis and intestinal inflammation. $\mathrm{HO}-1$ exerts a protective effect, which is partially correlated with the regulation of $\mathrm{NF}-\mathrm{kB}$. Online address: http://www.molmed.org
\end{abstract}

doi: $10.2119 / \mathrm{molmed} .2017 .00078$

\section{INTRODUCTION}

Cholestasis caused by disordered bile secretion and excretion is common in many liver diseases. Bile duct ligation (BDL)-treated rats exhibit cholestasis, increased oxidative stress and liver fibrosis, which ultimately lead to liver cirrhosis (1). The BDL rat model has been widely used to study cholestatic liver injury and the associated oxidative stress and fibrogenesis (2). Bile acids can also modulate the microbiome, and the absence of bile in the gut leads to mucosal injury, as demonstrated by increased intestinal permeability and increased bacterial translocation $(3,4)$. The interaction between the microbiome and the host liver is of particular interest in cholestatic liver disease, in which bile acids have been shown to both change the composition of the microbiome and impair intestinal integrity and barrier function $(5,6)$. Increased levels of lipopolysaccharide (LPS) entering the liver through the portal vein induce recruitment and activation of inflammatory cells and production of proinflammatory cytokines, causing the

Address correspondence to Zhijun Duan, Department of Gastroenterology, First Affiliated Hospital of Dalian Medical University, Dalian 11601 1, China. Phone: + 86-18098875588;

E-mail: cathydoctor@sina.com.

Submitted May 3, 2017; Accepted for Publication August 1, 2017; Published Online

(www.molmed.org) August 9, 2017.

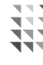

มีพ

Feinstein Institute

for Medical Research

Northwell Health

second hit to the liver and aggravating intestinal injury $(7,8)$. Therefore, the interactions between the gut microbiome, the intestinal barrier and the liver appear to have a key role in the pathogenesis of cholestatic liver disease, and the recovery of intestinal barrier function is crucial to block the progression of the disease.

The intestine epithelium forms a physical barrier between the lumen and the intestinal mucosa. Connections between intestinal epithelial cells include tight junctions (TJs), gap junctions, adhesion junctions and desmosomes. TJs maintain the permeability and integrity of the intestine; alterations in TJ protein expression have been found to contribute to impaired barrier function in many gastrointestinal and liver diseases, such as alcoholic liver disease, cirrhosis and inflammatory bowel disease (IBD) (9).

Heme oxygenase-1 (HO-1) is a stressinducible enzyme that exerts antioxidant, 
antiinflammatory and cytoprotective effects through its by-products including carbon monoxide (CO), biliverdin/bilirubin and free iron $(10,11)$. Previous reports have shown that induction of $\mathrm{HO}-1$ expression contributes to protection against liver damage in various liver diseases such as acute liver injury, alcoholic liver disease, liver fibrosis and ischemia/reperfusion injury (12-15). Encouragingly, activation of HO-1 counteracts LPS-induced inflammatory and oxidative damage (16) and can also alleviate intestinal ischemia-reperfusion injury (17). Our previous study found that endogenous activation of $\mathrm{HO}-1$ by cobalt protoporphyrin (CoPP) could suppress immune liver fibrosis (18), and another of our experiments showed that inhibiting $\mathrm{HO}-1$ activity could be helpful in ameliorating BDL-induced liver cirrhosis (19). Hence, HO-1 may exert diverse effects on the progression of liver disease, and the protective effect of HO-1 overexpression may be restricted to a narrow threshold. Because of the diverse effect of HO-1, we examined its effect in the early stage of liver fibrosis, mainly regarding the intestinal barrier this time, and the results showed that $\mathrm{HO}-1 / \mathrm{CO}$ has potential clinical applications for the treatment of intestinal dysfunction.

$\mathrm{CO}$ is a small gaseous molecule with antiinflammatory and antimicrobial properties. It is well established that both endogenous and exogenous $\mathrm{CO}$ can suppress inflammation, protect endothelial cells and improve microcirculation (20). CO-releasing molecule 2 (CORM-2) $\left[\mathrm{Ru}(\mathrm{CO})_{3} \mathrm{Cl}_{2}\right]_{2}$, one of the class of newly synthesized transition metal carbonyls, can release $\mathrm{CO}$ when it is dissolved in dimethyl sulfoxide (DMSO) (21). The inhibitory effect of $\mathrm{CO}$ and CORMs on cytokine-induced changes in the in-testinal epithelium might also contribute to their beneficial effect in acute and chronic gastrointestinal inflammatory conditions such as IBD $(22,23)$. It has been reported that CORM-2 exerts a protective effect on the barrier function of intestinal epithelial cells (24). In septic rats, it can also reduce intestinal epithelial TJ damage, protect barrier function and reduce mortality (25). However, little is known about the protective effect of HO-1/CORM-2 on the intestinal barrier in the early stage of cholestatic liver fibrosis. In this study, we mainly investigated the changes in the intestinal barrier in BDL-induced early cholestatic liver fibrosis and the protective effects of HO-1 overexpression and CORM2. We also constructed stable Caco- 2 cell lines that overexpressed HO-1 or had their HO-1 expression knocked down to elucidate further the protective effect of HO-1 and the possible mechanism of that effect.

\section{MATERIALS AND METHODS}

\section{Chemicals and Reagents}

LPS, CoPP, zinc protoporphyrin (ZnPP) and CORM-2 were purchased from Sigma-Aldrich. JSH-23 was obtained from Selleck Chemicals. CORM-2 and JSH-23 were dissolved in DMSO, then diluted to the required concentrations. Lipofectamine 2000 was obtained from Invitrogen. Anti-ZO-1 polyclonal antibody (Proteintech), anti-claudin-1 polyclonal antibody (Abnova), anti-occludin polyclonal antibody (Abcam), anti-Toll-like receptor 4 (TLR4) monoclonal antibody (Santa Cruz Biotechnology), anti-NF-кB p65 and Proliferation Cell Nuclear Antigen (PCNA) monoclonal antibodies (Cell Signaling Technology) and peroxidase-conjugated goat anti-rabbit or anti-mouse secondary antibody (ZSGB-BIO) were used. Alexa Fluor 594 anti-rabbit Immunoglobulin G (IgG) and Alexa Fluor 488 anti-mouse IgG were from Proteintech. Puromycin was obtained from Beijing Solarbio Science \& Technology Co., Ltd.

\section{Cell Culture}

Human colonic epithelial cells (Caco-2) were purchased from the American Type Culture Collection (ATCC) and cultured in Dulbecco's modified Eagle's medium with high glucose (DMEM, Gibco), supplemented with $10 \%$ fetal bovine serum (FBS). Cells were cultured at $37^{\circ} \mathrm{C}$ with $5 \% \mathrm{CO}_{2}$. When the cells reached approximately $70 \%-80 \%$ confluency, they were dissociated with trypsin and subcultured. To detect the phosphorylation levels and nuclear translocation of NF- $\kappa \mathrm{B}$, we treated Caco-2 cells with or without
JSH-23 $(30 \mu \mathrm{mol} / \mathrm{L})$ for $1 \mathrm{~h}$ and stimulated them with $10 \mu \mathrm{g} / \mathrm{mL}$ LPS for $24 \mathrm{~h}$.

\section{Animal Model (BDL and Grouping)}

Animal experiments were carried out in strict accordance with the recommendations in the guide of the Animal Care and Use Committee of Dalian Medical University (Liaoning Province, China), and the protocol was approved by the institutional Ethics Committee (approval ID L20160221). Sixty male Sprague-Dawley rats, weighing 200-220 g, were obtained from the Laboratory Animal Center of Dalian Medical University and were divided randomly into five groups: Sham $(\mathrm{n}=8)$, BDL $(\mathrm{n}=14)$, cobalt protoporphyrin (CoPP, $\mathrm{n}=14)$, zinc protoporphyrin $(\mathrm{ZnPP}, \mathrm{n}=10)$ and CORM-2 $(\mathrm{n}=14)$. The rats were fed ad libitum and housed for $3 \mathrm{~d}$ before any experimental protocols were conducted. Cholestatic liver injury was induced by BDL. Briefly, the bile duct was isolated and loosely double ligated with a 3-0 silk suture as previously described (26). Rats in the Sham group underwent laparotomy and had the bile duct isolated but not ligated. The other four groups underwent BDL surgery. Two weeks after BDL surgery, the animals were treated by intraperitoneal injection with CoPP or $\mathrm{ZnPP}$ (5 mg/kg body weight) or CORM-2 (30 mg/kg body weight) every other day for a total of three injections. $\mathrm{ZnPP}$ and CoPP were first dissolved in $0.2 \mathrm{~mol} / \mathrm{L}$ $\mathrm{NaOH}$ (pH 7.4), then diluted in $0.85 \%$ $\mathrm{NaCl}$ for a final concentration of $1 \mathrm{mg} / \mathrm{mL}$ as previously described, and the compounds were used for inhibiting and inducing $\mathrm{HO}-1$ expression, respectively (27). For the Sham and BDL groups, rats received an intraperitoneal injection of saline. At the end of the treatment, the number of rats was reduced to 42 : Sham, $\mathrm{n}=6$; $\mathrm{BDL}, \mathrm{n}=10 ; \mathrm{CoPP}, \mathrm{n}=10 ; \mathrm{ZnPP}$, $\mathrm{n}=6$; and CORM-2, $\mathrm{n}=10$.

\section{Sample Collection and Examination}

Blood samples were collected from the abdominal vein and centrifuged for biochemical and enzyme-linked immunosorbent assay (ELISA) measurement. Serum levels of alanine 
aminotransferase (ALT), aspartate transaminase (AST), total bile acid (TBA), $\gamma$-Glutamyl Transpeptidase $(\gamma-\mathrm{GT})$ and alkaline phosphatase (AKP) were measured. Segments of colon and liver tissue were harvested, some of which were Pxed in 4\% paraformaldehyde and used for histological examination. Another portion was immediately stored at $-80^{\circ} \mathrm{C}$.

\section{Histological Scoring}

Liver and intestinal tissues from each animal were stained with hematoxylin and eosin (H\&E). Liver fibrosis was graded as follows: 0 = no fibrosis; 1 = enlarged, fibrotic portal tracts; 2 = periportal or portal-portal septa but intact architecture; 3 = fibrosis with architectural distortion but no obvious cirrhosis; 4 = probable or definite cirrhosis.

Intestinal mucosal injuries were graded according to the scoring system described by Chiu (28): 0 = normal mucosa; 1 = mucosal degeneration with extended subepithelial space; 2 = intestinal villus epithelium raised and more extended subepithelial space; 3 = intestinal villus epithelium deciduation; 4 = intestinal villus epithelium shedding, only lamina propria; 5 = severe degeneration and mucosal digestion with disintegration of lamina propria, bleeding and ulcers.

\section{Construction of Stable HO- 1 Overexpression and Knockdown Cell Lines}

An FUGW expression vector carrying the human HO-1 gene was constructed. Cells were transfected with either an FUGW-HO-1 plasmid or an FUGW vector control plasmid. Short hairpin RNA (shRNA) targeting the $\mathrm{HO}-1$ gene (5'-CCGGACAGTTGCTGTA GGGCTTTATCTCGAGATAAAG CCCTACAGCAACTGTTTTTTG-3') or a scrambled control shRNA was cloned into a pLKO.1 vector to construct an HO-1 knockdown cell line. Lentiviral transductions with the HO-1 gene or $\mathrm{HO}-1$ shRNA were conducted in Caco-2 cells, and the cells were selected with puromycin $(4 \mu \mathrm{g} / \mathrm{mL})$ for $3 \mathrm{~d}$ and cultured at $1 \mu \mathrm{g} / \mathrm{mL}$ of puromycin.
Successful overexpression or silencing of HO-1 in stable cells was confirmed by real-time Polymerase Chain Reaction (PCR) and Western blot.

\section{RT-qPCR}

Total RNA from Caco-2 cells was extracted using TRIzol Reagent (Transgene Biotech) according to the manufacturer's instructions. Reverse transcription (RT) was performed on $1 \mu \mathrm{g}$ of total RNA using random primers (PrimeScript ${ }^{\mathrm{TM}}$ RT Reagent Kit, Takara). PCR was performed using SYBR Fast qPCR Mix (TaKaRa). The HO-1 and $\beta$-actin primer sequences were as follows.

HO-1: Forward 5'-TCCGATGGGTCCTTACACTC-3', Reverse 5'-AA GGAAGCCAGCCAAGAGA-3'.

$\beta$-actin: Forward 5'-AGCCTCAAGA TCATCAGCAATG-3',

Reverse 5'-TGTGGTCATGAGTCCTT CCACG-3'. The PCR cycling conditions consisted of one cycle of $95^{\circ} \mathrm{C}$ for $10 \mathrm{~min}$ for denaturing followed by 40 cycles of $95^{\circ} \mathrm{C}$ for $15 \mathrm{~s}$ and $60^{\circ} \mathrm{C}$ for $1 \mathrm{~min}$. The messenger RNA (mRNA) expression of target genes was normalized to the expression of $\beta$-actin.

\section{Western Blot Analysis}

Proteins were extracted from cultured cells or rat tissues using lysis buffer, and their concentrations were determined by the BCA method (BCA kit, Trans). Equal amounts of protein were resolved by sodium dodecyl sulfate polyacrylamide gel electrophoresis (SDS-PAGE), and then the proteins were transferred onto polyvinylidene difluoride (PVDF) membranes, which were then blocked and probed with the appropriate antibodies at $4^{\circ} \mathrm{C}$ overnight. Subsequently, the membranes were incubated with HRP-conjugated secondary antibody (1:8000) for $2 \mathrm{~h}$ at room temperature. Blots were visualized using a chemiluminescent kit (Advasta). GAPDH and $\beta$-actin were used as control proteins, and the relative intensity of protein bands were analyzed by the Quantity One program (Bio-Rad Laboratories).

The proteins in the nucleus and cytoplasm were extracted using a nuclear and cytoplasmic protein extraction kit (KeyGene Biotec), following the instructions issued with the kit. Then, as described above, the nuclear and cytoplasmic protein were analyzed with NF-кB p65 antibody. $\beta$-actin and Lamin $\mathrm{A} / \mathrm{C}$ were used as loading controls for protein in the cytoplasm and nucleus, respectively.

\section{Immunofluorescence Staining}

The cells, after being grown on cover glasses, were fixed with $4 \%$ paraformaldehyde for $20 \mathrm{~min}$ and treated with $0.3 \%$ Triton $\times 100$, then stained with the anti-NF- $\kappa$ B p65 antibody (1:100) at $4^{\circ} \mathrm{C}$ overnight. The cells were detected with Alexa Fluor 594 anti-rabbit IgG. The coverslips were counterstained with 4',6-diamidino-2-phenylindole (DAPI) and imaged using a confocal laser scanning microscope (Olympus). Untreated Caco-2 cells were used as a normal control.

For the intestine tissue staining, the deparaffinized sections were microwaved in citrate buffer and then incubated with rabbit monoclonal anti-p65 and mouse monoclonal anti-TLR4 antibodies, followed by incubation with Alexa Fluor 594 goat anti-rabbit IgG and Alexa Fluor 488 goat anti-mouse IgG. The nuclei were stained with DAPI, and the tissue was examined under a fluorescence microscope.

\section{Enzyme-Linked Immunosorbent Assay (ELISA)}

Serum endotoxin, diamine oxidase (DAO) and D-Lactic acid (D-Lac) were measured by ELISA according to the manufacturer's instructions (Lengton Biological Technology Co.). Intestinal TNF- $\alpha$ and IL- 6 expression levels were measured as well. All standards and samples were run in duplicate.

\section{Statistical Analysis}

Statistical analysis was carried out using SPSS 16.0 for Windows. Data are presented as the mean $\pm S D$, and the significance of differences was assessed by analysis of variance (ANOVA) or Student $t$ test. Differences were considered significant when the $P$ value was less than 0.05 . 
All supplementary materials are available online at www.molmed.org.

\section{RESULTS}

\section{COPP and CORM-2 Alleviated Liver and Intestinal Mucosa Injury in BDL Rats}

We first evaluated the effects of BDL on liver and intestinal mucosa injury. The serum levels of ALT, AST, TBA, AKP and $\gamma$-GT in the BDL group were significantly higher than those in the Sham group $(P<0.05$, Supplementary Figure S1). H\&E staining verified that BDL rats exhibited different degrees of fibrosis (80\% grade 2 ), while the Sham group exhibited normal hepatic architecture or only the slightest injury (Supplementary Figure S2). In addition to the liver injury, the intestinal mucosa exhibited various degrees of damage in BDL rats. The BDL group showed epithelial cell layer degeneration, necrosis and shedding; in addition, the intestinal villi were not well arranged, and there was obvious infiltration by inflammatory cells (Supplementary Figure S3). In the CoPP and CORM-2 treatment groups, the levels of serum ALT, AKP, $\gamma$-GT and TBA were obviously decreased compared with those of the BDL group, and the liver fibrosis grade was also lower (Supplementary Figure S2 and Table 1). The intestinal mucosa injury was evaluated with the scoring standards described by Chiu (28). In the CoPP and CORM-2 groups, the degree of intestinal mucosa injury was significantly reduced, showing no obvious signs except edema and falling mostly in Grade 0 to Grade 1 (details provided in Table 2).

We also evaluated the effects of BDL on endotoxin, DAO and D-Lac levels in serum, as these proteins are important indicators of intestinal permeability and integrity. The results are reported in Figure 1. D-Lac and DAO in the BDL group were significantly increased compared with the Sham group, CORM-2 and CoPP treatment both decreased the levels of endotoxin and D-Lac, whereas the $\mathrm{ZnPP}$ group had no significant difference from the BDL group. These findings indicate that CoPP and CORM-2 played

Table 1. Pathological grading of liver fibrosis in SD rats.

\begin{tabular}{lcllll}
\hline & $n$ & Grade 0 & Grade 1 & Grade 2 & Grade 3 \\
\hline Sham & 6 & $4(66.7 \%)$ & $2(33.3 \%)$ & 0 & 0 \\
BDL & 10 & 0 & $1(10 \%)$ & $8(80 \%)$ & $1(10 \%)$ \\
CORM-2 & 10 & $2(20 \%)$ & $7(70 \%)$ & $1(10 \%)$ & 0 \\
CoPP & 10 & 0 & $5(50 \%)$ & $5(50 \%)$ & 0 \\
ZnPP & 6 & 0 & $1(16.7 \%)$ & $5(83.3 \%)$ & 0 \\
\hline
\end{tabular}

Table 2. Judgment of intestinal mucosal injury in SD rats.

\begin{tabular}{lrllll}
\hline & $\mathrm{n}$ & Grade 0 & Grade 1 & Grade 2 & Grade 3 \\
\hline Sham & 6 & $4(66.7 \%)$ & $1(16.6 \%)$ & $1(16.7 \%)$ & 0 \\
BDL & 10 & 0 & $1(10 \%)$ & $4(40 \%)$ & $5(50 \%)$ \\
CORM-2 & 10 & $3(30 \%)$ & $6(60 \%)$ & $1(10 \%)$ & 0 \\
CoPP & 10 & $2(20 \%)$ & $5(50 \%)$ & $2(20 \%)$ & $1(10 \%)$ \\
ZnPP & 6 & $1(16.7 \%)$ & $2(33.3 \%)$ & $3(50 \%)$ & 0 \\
\hline
\end{tabular}

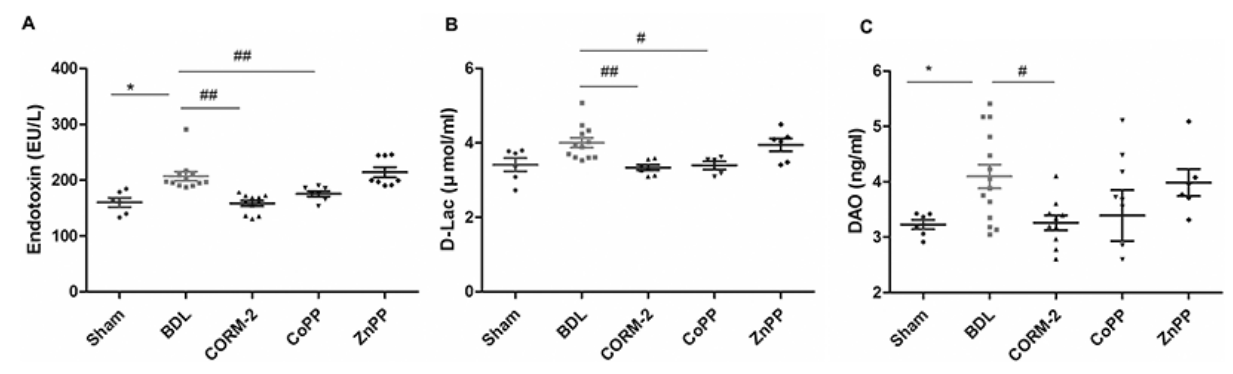

Figure 1. ELISA assay for markers of intestinal injury in control (Sham) and BDL rats in the absence or presence of HO-1 activators. (A) serum endotoxin; (B) serum D-Lac; (C) serum $\mathrm{DAO}$. Serum endotoxin, D-Lac and DAO were significantly increased in the BDL group compared with the Sham group, " $P<0.05$. CORM-2 or CoPP treatment decreased the levels of endotoxin and D-Lac, ${ }^{\#} P<0.05,{ }^{\# \#} P<0.01$ compared with the BDL group.

a role in protection against BDL-induced intestinal mucosa injury.

\section{Effect of COPP and CORM-2 on Tight Junction, Cell Apoptosis, PCNA and TLR4/NF- $\kappa$ B Expression in Intestinal Tissue from Sham/BDL Rats}

The ELISA results in Figure 1 show that in CoPP- and CORM-2-treated BDL rats, intestinal barrier dysfunction was relieved. Intestinal dysfunction is mainly manifested as the disruption of TJs and intestinal epithelium. Activation of $\mathrm{HO}-1$ counteracts LPS-induced intestinal damage. Therefore, we measured the levels of intestinal epithelium apoptosis, proliferation and TJ-related protein (ZO-1, claudin-1) expression as well as TLR4 and $\mathrm{NF}-\kappa \mathrm{B}$ in intestinal tissue from Sham/BDL rats. The results are displayed in Figure 2.
The expression levels of HO-1, TLR4 and NF- $\kappa$ B p65 were notably increased in the BDL group compared with the Sham group (Figure 2A, C), whereas ZO-1 and claudin-1 were significantly reduced (Figure 2B). Meanwhile, the expression levels of the apoptosis-related protein caspase 3 and its active form cleaved caspase 3 were significantly elevated. Conversely, PCNA expression in the BDL group was decreased (Figure 2D).

In the CoPP and CORM-2 groups, cleaved caspase 3 and TLR4 expression levels were decreased, while PCNA protein increased, suggesting that CoPP and CORM-2 could both reduce cell apoptosis and promote cell proliferation. In the CORM-2 treatment group, the activation

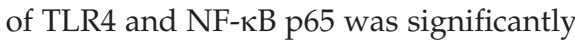
inhibited. More importantly, in the CoPP 


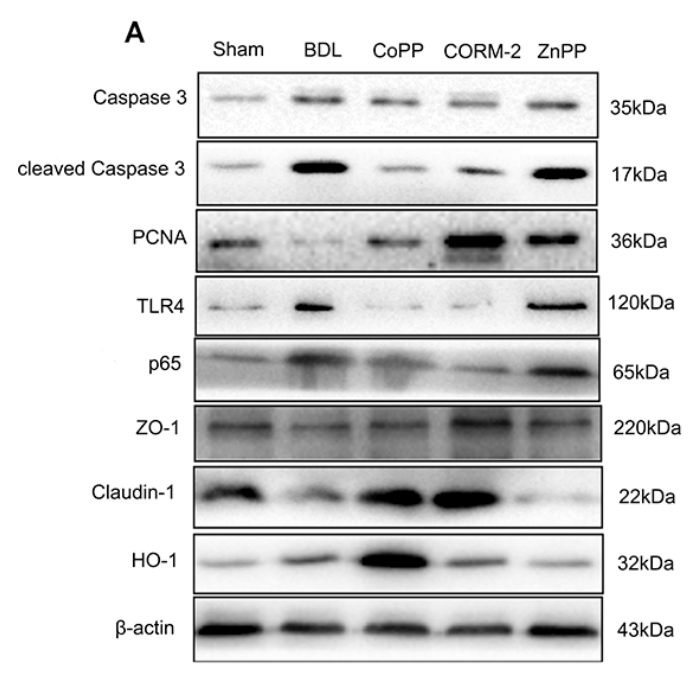

B

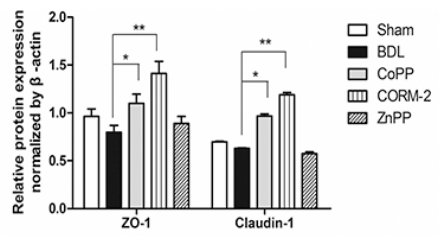

C

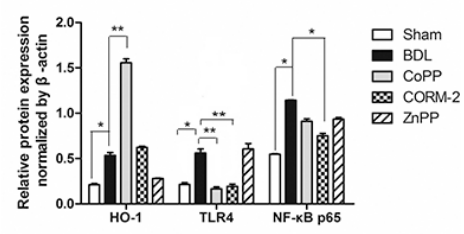

D

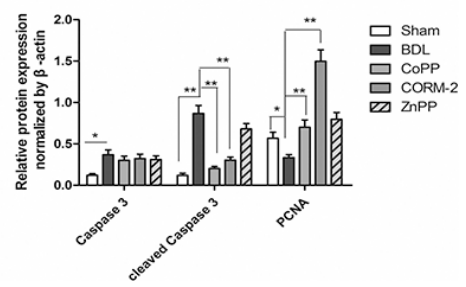

Figure 2. Effects of CoPP and CORM-2 on tight junctions, cell apoptosis, PCNA and TLR4/NF-kB expression in intestinal tissue from Sham/BDL rats. (A) Western blot images; (B) the relative expression of ZO-1 and occludin. The expression levels of the TJ proteins ZO-1 and claudin- 1 expression were significantly increased in the COPP and CORM-2 groups compared with the BDL group; (C) HO-1, TLR4 and NF-KB p65 were notably increased in BDL group compared with the Sham; (D) caspase 3, cleaved caspase 3 and PCNA expression. Data are presented as the mean \pm SD from three independent experiments, ${ }^{*} P<0.05,{ }^{* *} P<0.01$.

and CORM-2 groups, the expression levels of the TJ proteins ZO- 1 and claudin- 1 expression were significantly increased compared with the BDL group (Figure 2A, B). All these findings indicated that CORM-2 and CoPP treatments protected the tight junction, inhibited cell apoptosis and promoted intestinal epithelial cell proliferation in addition to decreasing the expression of TLR4 and NF-kB.

\section{COPP and CORM-2 Decreased Intestinal TNF- $\alpha$ and IL-6 Expression}

We also studied the development of intestinal inflammation in BDL rats. ELISA results for TNF- $\alpha$ and IL- 6 in intestinal tissue also confirmed that BDL was accompanied by intestinal barrier dysfunction and increased inflammation (IL-6 increased). There was also a trend toward increased TNF- $\alpha$ in BDL rats ( $P=0.078$, Figure 3$)$. CORM- 2 or CoPP treatment protected intestinal barrier function and decreased IL-6 production
(Figure 3). HO-1 increased ZO-1 and occludin expression in vitro.

The results shown in Figure 2 demonstrate that in CoPP-treated BDL rats HO-1 expression was notably elevated, which also improved the epithelial tight junctions. ZO-1, occludin and claudin- 1 are important indicators of TJs. To further verify the role of HO- 1 in the protection

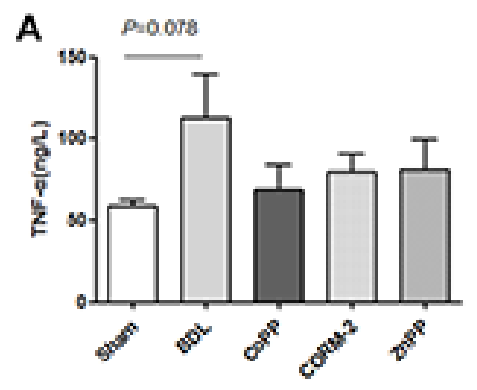

of the intestinal barrier, we constructed a stable Caco-2 cell line overexpressing $\mathrm{HO}$ and another with HO-1 expression knocked down. qPCR results showed that HO-1 was significantly up-regulated in the HO-1 overexpression cells (Figure 4A) and remarkably decreased in the sh-HO-1 transfection cells (Figure 4C). Western blot results confirmed that HO- 1 protein expression followed the same pattern. More importantly, the level of HO-1 appeared to influence the expression of $\mathrm{TJ}$ proteins. As shown in Figure 4B, ZO-1, occludin and claudin-1 expression levels were significantly increased in the HO-1 overexpression group. Meanwhile, sh-HO-1 cells showed reduced levels of ZO- 1 and occludin compared with the sh-control group, but claudin-1 protein expression showed no obvious difference (Figure 4D).

\section{HO- 1 Protected against Intestinal Barrier Damage by Inhibiting Activation of NF- $\kappa$ B}

As observed in the animal experiment, HO-1 overexpression can inhibit TLR4 and NF-кB expression; thus, we analyzed the effect of $\mathrm{HO}-1$ on the cellular distribution of NF-kB. The cytoplasmic and nuclear proteins were separated, and Western blot results showed that LPS significantly increased nuclear NF-кB p65 protein expression and decreased cytoplasmic p65 expression compared with the values in the control group. Moreover, HO-1 overexpression (by transfection with HO-1 plasmids) decreased the level of NF-кB (p65) in the

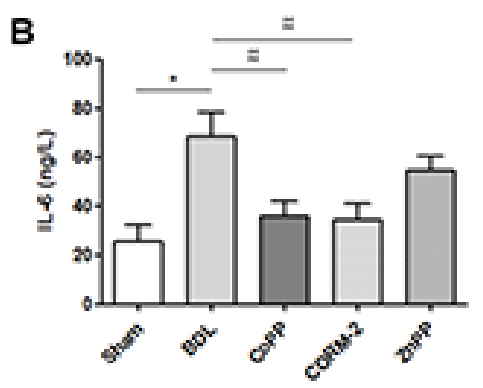

Figure 3. Intestinal TNF- $\alpha$ and IL-6 detection by ELISA assay in Sham/BDL rats. (A) TNF- $\alpha$; (B) IL-6. Intestinal IL-6 expression was significantly increased in the BDL group compared with the Sham group, and CORM-2 or CoPP treatment decreased intestinal IL-6 expression. ${ }^{*} P<0.05$ compared with the Sham; ${ }^{*} P<0.05,{ }^{\#} P<0.01$ compared with the BDL group. 
A

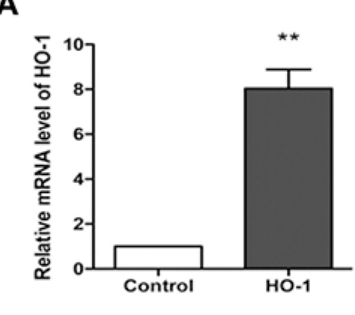

C

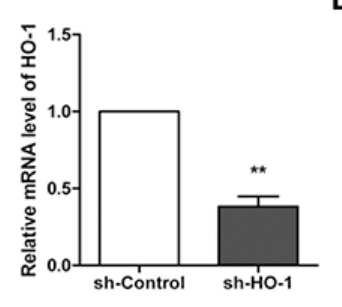

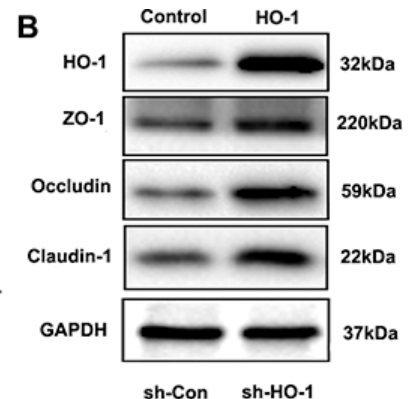

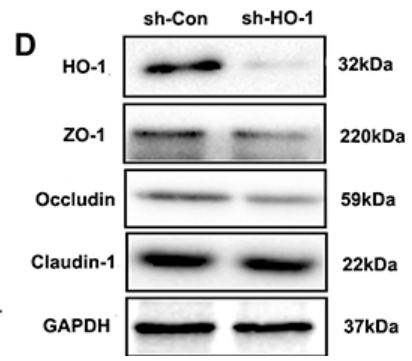

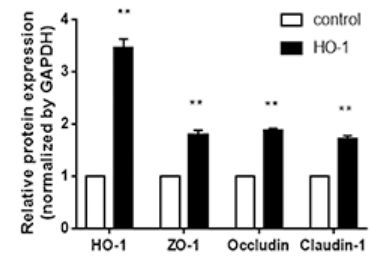

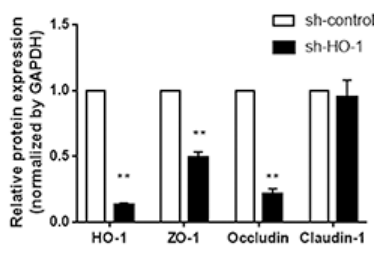

Figure 4. HO-1 and TJ protein expression in stable Caco-2 cells. (A) q-PCR results confirmed that $\mathrm{HO}-1$ was overexpressed; (B) Western blot results verified that $\mathrm{HO}-1$ protein was up-regulated in HO-1-transfected Caco-2 cells; meanwhile, ZO-1, occludin and claudin-1 expression levels were increased compared with the control. (C, D) q-PCR and Western blot results from the sh-HO-1 group. $\mathrm{HO}-1$ expression was significantly decreased in the sh-HO-1 group; ZO-1 and occludin were down-regulated as well. Data are presented as the mean \pm SD from three independent experiments. " $P<0.01$ compared with the corresponding control.

nucleus, while in sh-HO-1 Caco-2 cells, the nuclear p65 level was significantly elevated (Figure 5A, B). Immunofluorescence staining further confirmed that, in the control group, p65 protein was mainly localized to the cytoplasm. However, when LPS stimulation was applied, strong p65 staining in the nucleus was observed. In the HO-1 overexpression cells, in contrast, nuclear accumulation of p65 in response to LPS stimulation was significantly reduced (Figure 5C), and the reverse was true in sh-HO- 1 caco- 2 cells. The results indicate that HO-1 overexpression may reduce intestinal barrier damage by inhibiting the activation of NF- $\mathrm{NB}$.

\section{HO- 1 Increased Occludin and Inhibited Phospho-NF-אB p65 Protein Expression}

To further elucidate the protective mechanism of HO-1, we used the specific

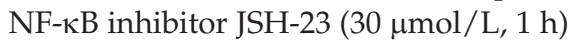
to treat the Caco-2 cells before LPS stimulation, and we then measured occludin protein expression. The results showed that JSH-23 significantly increased occludin protein expression in both the $\mathrm{HO}-1$ overexpression and sh-HO-1 groups compared with their respective controls (Figure 6A, B). More importantly, when JSH-23 + LPS stimulation was applied to HO-1 overexpressed Caco-2 cells, occludin expression was notably higher than that in the sh-HO-1 group. Previous results (Figure 5) had confirmed that HO-1 up-regulation could inhibit NF- $\kappa \mathrm{B}$ activation; hence, we compared phospho-NF-кB p65 (p-p65) protein expression. As shown in Figure 6C, the expression of $\mathrm{p}-\mathrm{p} 65$ protein in sh-HO-1 Caco-2 cells was obviously higher than that in the HO-1 overexpression group. In addition, p-p65 expression was significantly increased under LPS stimulation in sh-HO-1 Caco- 2 cells. Therefore, we hypothesized that the loss of HO-1 could promote the expression of p-p65 and that $\mathrm{HO}-1$ overexpression could inhibit NF-кB p65 phosphorylation and increase occludin expression, which would further verify that $\mathrm{HO}-1$ protects intestinal TJs by inhibiting NF-אB activation.

\section{Immunofluorescent Staining Results for NF- $\mathrm{kB}$ p65 and TLR4 in Intestinal Tissue from Sham/BDL Rats}

To further confirm that the protective effect of HO-1/CORM-2 was correlated with NF- $\kappa$ B, we used immunofluorescent staining to detect p65 and TLR4 expression in vivo. p65 was mainly located in the cytoplasm of the intestinal epithelial cell in Sham group, while in the BDL rats, nuclear p65 expression was significantly increased. However, there was less nuclear translocation in CoPP/ CORM-2-treated BDL rats than in BDL rats (Supplementary Figure S4), demonstrating that CoPP/CORM-2 could inhibit NF-kB p65 nuclear translocation. TLR4 immunofluorescent staining results also verified that TLR4 expression was much higher in BDL than in Sham rats, but it was reduced in BDL rats treated with CoPP/CORM-2 (Supplementary Figure S5). Based on these results, we concluded that HO-1/CORM-2 was closely related to TLR4/NF-кB inhibition.

\section{DISCUSSION}

Cholestasis is a common clinical symptom characterized as an increase in bile acids in blood and liver tissue. A high concentration of bile acids, especially the hydrophobic bile acids, is particularly toxic and promotes hepatocyte damage, liver fibrosis, cirrhosis and hepatocellular carcinoma under cholestatic conditions $(29,30)$. Notably, the reduced flow of bile acids into the small intestine facilitates the movement of flora and bacterial endotoxins from the portal vein into the liver and causes disruption of the intestinal epithelial barrier (31-33). Our previous study found that chronic liver injury patients also displayed intestinal barrier dysfunction, manifesting as significantly elevated serum endotoxin, DAO and D-Lac compared with normal controls (data not shown). In this study, we used the improved BDL model (loosely double-ligated) in rats to mimic cholestatic 
A
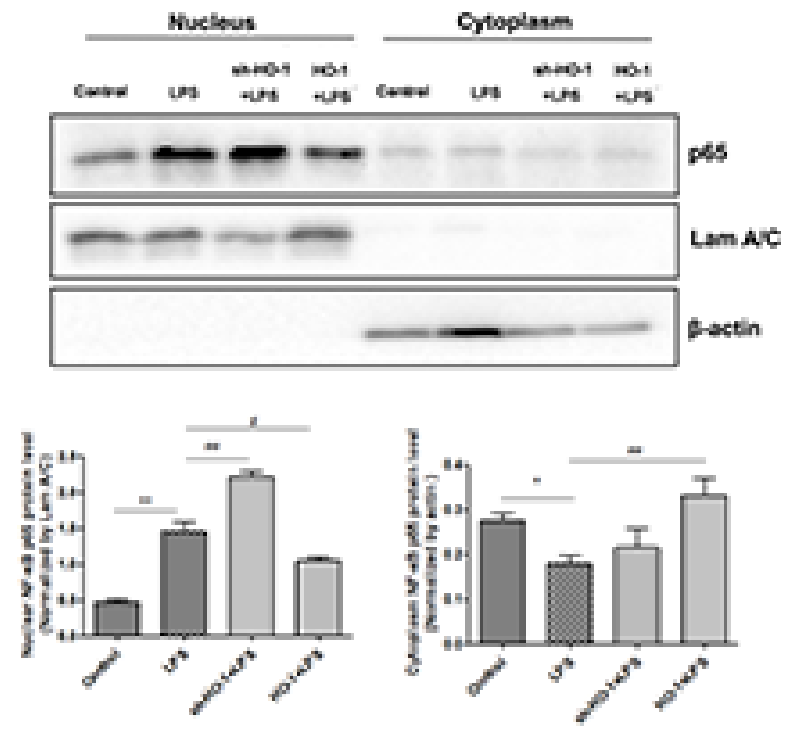

B
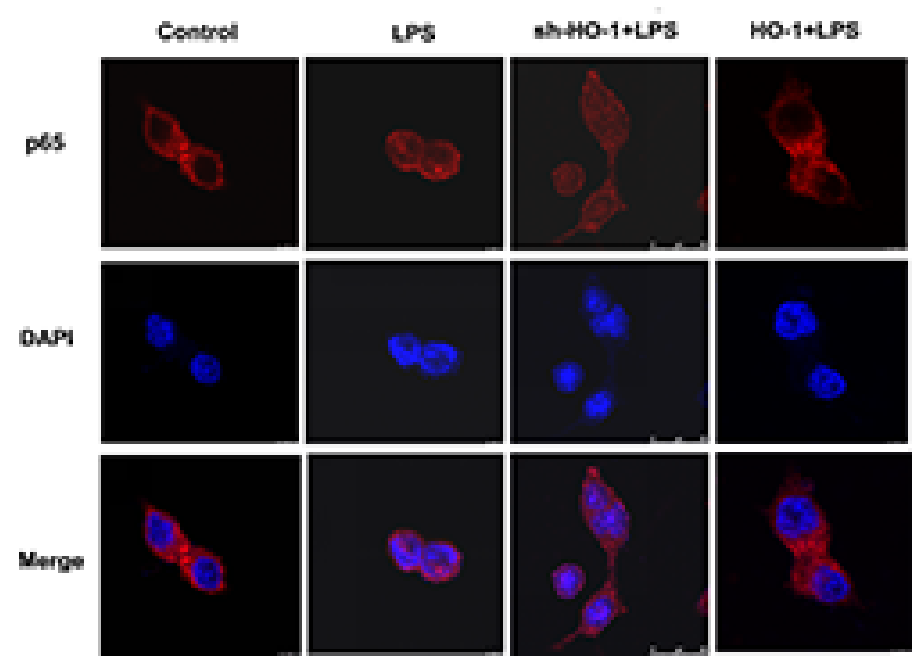

Figure 5. HO- 1 overexpression inhibits NF- $\mathrm{kB}$ nuclear translocation. Cytoplasmic and nuclear proteins were extracted, and NF-кB p65 was determined by Western blot analysis $(A, B)$. The expression levels of $\beta$-actin and Lamin $A / C$ were used as loading controls. " $P<0.05$, " $P<0.01$ compared with control group; ${ }^{\#} P<0.05,{ }^{\# \#} P<0.01$ compared with LPS treatment group. (C) Immunofluorescence staining results for NF-kB p65. Red is p65; blue is DAPI (nuclei).

liver injury and to observe the protective effects of $\mathrm{HO}-1 / \mathrm{CO}$ on intestinal barrier function.

The increased serum biochemical parameters confirmed that there was liver injury in BDL rats. H\&E staining and ELISA results verified that BDL rats exhibited varying degrees not only of liver fibrosis (80\% in Grade 2) but also of intestinal barrier disruption. The fact that serum endotoxin, D-Lac and DAO were significantly increased demonstrates this. The presence of bile in the gut is essential for the maintenance of intestinal mucosal barrier function; bile salt deficiency can lead to intestinal mucosal atrophy and intestinal mucosa barrier dysfunction (34). The elevated serum endotoxin and D-lac suggested that intestinal permeability increased and the intestinal barrier was damaged. The pathological results also confirmed the presence of intestinal mucosa disruption in BDL rats.

$\mathrm{HO}-1$, a heat-shock protein, has been implicated in a cytoprotective mechanism to protect tissues from oxidative damage (35). Induction of HO-1 expression contributes to protection against liver damage in various liver diseases $(36,37)$. We found that up-regulation of HO-1 could alleviate liver damage in BDL rats, which is in accordance with the findings of Barikbin et al. In their study, induction of HO-1 interfered with chronic inflammation and prevented progression of liver fibrosis in Mdr2 knockout mice (36). However, its protective effects in liver fibrosis have been disputed. Some studies have shown that $\mathrm{HO}-1$ overexpression increases liver injury in rats under conditions of experimental chronic cholestasis (38). Our previous study of liver cirrhosis also demonstrated that lower HO-1 expression could mitigate end-stage liver cirrhosis by reducing iron accumulation (19). The reason why the above studies are different from this study could be that $\mathrm{HO}-1$ plays diverse roles in different stages during the progression of liver fibrosis. In early stages of liver fibrosis, the liver mainly expressed in fatty degeneration of injured liver cells and inflammatory cell infiltration, while the hepatic blood circulation and portal vein pressure were not affected. In this study, the improved BDL model induced the early stage of liver fibrosis; therefore, inducing $\mathrm{HO}-1$ could have a protective effect against hepatic injury, but it could increase liver injury in the end stages of fibrosis via hemodynamic disturbances.

The role of intestinal permeability and inflammation has received recent attention, and it has been shown that, in patients with portal hypertension, the gut barrier function is impaired, leading to increased gut "leakiness" (39). HO-1 plays an important role in improving intestinal barrier function. In a rat liver transplantation model, elevation of $\mathrm{HO}-1$ mitigates intestinal ischemia-reperfusion injury and restores TJ function (17), and 

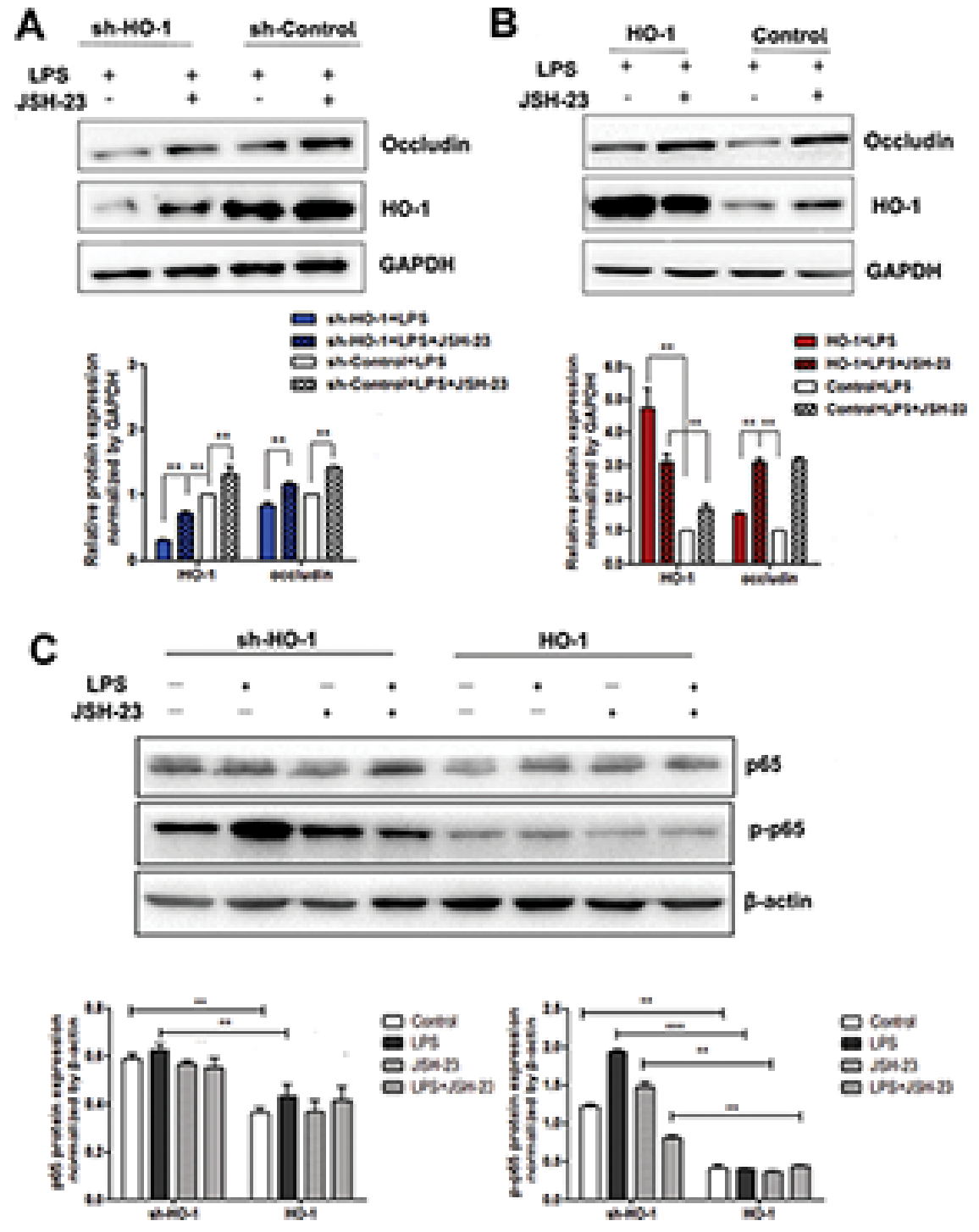

Figure 6. Effect of JSH-23 on occludin, $\mathrm{p} 65$ and p-p65 protein expression in Caco-2 cells in which $\mathrm{HO}-1$ was overexpressed or knocked down. (A) HO-1 and occludin protein expression in HO-1 knockdown (sh-HO-1) Caco-2 cells. (B) HO- 1 and occludin in $\mathrm{HO}-1$ overexpressed Caco-2 cells. (C) p65 and p-p65 protein expression. The intensity of the band was quantified using the values obtained from the immunodetection of $\beta$-actin or GAPDH. Data are presented as the mean \pm SD from three independent experiments, ${ }^{*} P<0.05$, ${ }^{* *} P<0.01,{ }^{* * *} P<0.001$.

CORM-2 has also been reported to alleviate intestinal epithelial TJ damage in septic rats (25). Our study also found that CoPP could endogenously activate $\mathrm{HO}-1$ expression in BDL rats and reduce damage to the intestinal mucosa. At the same time, the decreased serum endotoxin, DAO and D-Lac levels also verified that CoPP and CORM-2 exert some protective effect against intestinal barrier disruption in BDL rats. A TJ is a fundamental junctional complex of adjacent epithelial cells and is composed of several protein families including transmembrane proteins (such as occludin and claudins) and peripheral proteins ( $\mathrm{ZO}$ protein). The TJ complex is critical for maintaining mucosal barrier integrity (40), and disruption of TJs is an important component of altered intestinal epithelial barrier function (41). Numerous previous studies have confirmed the role of $\mathrm{HO}-1$ in countering inflammation and oxidative stress. However, little is known about the effect of HO-1 on liver and intestinal injury in BDL-induced early-stage liver fibrosis. The present study demonstrated that up-regulation of $\mathrm{HO}-1$ or exogenous supplementation of $\mathrm{CO}$ by CORM-2 induced significant increases in ZO-1 and claudin-1 expression and reduced intestinal epithelial cell apoptosis and intestinal inflammation (intestine IL-6 expression decreased).

The LPS-activated TLR4 receptor complex results in downstream activation of the NF- $\kappa \mathrm{B}$ pathway and induction of proinflammatory cytokines and chemokines. Among those are TNF $\alpha$, IL- 6 and IL-1 $\beta$, which have been shown to disrupt the integrity of the TJ complex and increase intestinal barrier permeability $(42,43)$, thereby potentially amplifying gut leakiness and hastening the progress of liver disease. In our study, intestinal inflammation was relieved after CoPP or CORM-2 treatment; therefore, we speculated that $\mathrm{HO}-1$ exerts a protective effect on the intestinal barrier by preventing TJ damage and cell apoptosis, which are closely related to the TLR4/NF- $\mathrm{B}$ pathway.

To deeply explore the effects of HO-1 on the intestinal barrier and the mechanisms of those effects, we constructed stable Caco-2 cell lines with overexpression or knockdown of HO-1 and observed the expression change in the TJ protein occludin in response to our manipulation of HO-1. Occludin, recognized as a typical TJ protein, was closely correlated with the barrier function (44). Our results showed that occludin protein expression increased in $\mathrm{HO}-1-$ overexpressing Caco- 2 cells. In contrast, Caco-2 cells with HO-1 knockdown exhibited decreased expression of occludin, suggesting that HO-1 was closely related to $\mathrm{TJ}$.

It is well known that NF- $\kappa \mathrm{B}$ is important in terms of directing the transcription of many inflammatory genes following exposure to LPS (45). Data from other 
investigators have demonstrated that HO-1/CORM-2 can effectively inhibit activation of the NF- $\kappa$ B pathway $(46,47)$. In vivo research has shown that CoPP and CORM-2 treatment significantly inhibits the expression of TLR4 and NF- $\kappa \mathrm{B}$ compared with that of BDL rats, and immunofluorescent staining results in vivo have also verified that CoPP/CORM-2 inhibits TLR4 expression and p65 nuclear translocation. Therefore, inhibition of the NF-kB pathway may be the mechanism by which $\mathrm{HO}-1 / \mathrm{CO}$ protects the intestinal barrier. Under normal conditions, $\mathrm{NF}-\kappa \mathrm{B}$ is sequestered in the cytoplasm. Upon stimulation, such as with LPS or by activation of TLRs, $\mathrm{I} \kappa \mathrm{B} \alpha$ is degraded, resulting in the nuclear translocation of NF- $\kappa B$ (48). NF- $\kappa B$ binds to specific DNA sequences to initiate the transcription of inflammatory genes. Our results revealed that HO-1 suppressed the LPS-induced nuclear translocation of NF- $\kappa \mathrm{B}$ in vitro, which then inhibited the expression of proinflammatory cytokines, such as TNF- $\alpha$, IL-1 $\beta$ and IL-6.

As the protective effect of $\mathrm{HO}-1$ was correlated with NF- $\kappa$ B activation, we applied JSH-23, a specific inhibitor of NF- $\kappa \mathrm{B}$ transcriptional activity, before LPS stimulation and found that JSH-23 increased occludin protein expression not only in the HO-1 overexpression group but also in the sh-HO-1 group. The increased occludin protein demonstrated that inhibition of NF- $\mathrm{BB}$ transcription could alleviate inflammatory cytokine-induced intestinal barrier disruption. More importantly, in the shHO-1 group, p-p65 protein expression was significantly increased compared with the HO-1 overexpression group, suggesting that $\mathrm{HO}-1$ could inhibit NF-кB p65 phosphorylation and increase occludin expression, which further verified that $\mathrm{HO}-1$ protects intestinal TJs by inhibiting NF- $\kappa \mathrm{B}$ activation.

\section{CONCLUSION}

HO-1 played a dual and pivotal role in protection against liver and intestinal injury in BDL-induced early-stage liver fibrosis (Supplementary Figure S6). Our results demonstrate that high expression of HO-1 significantly improves intestinal epithelial barrier function, mainly through restoring TJ proteins, reducing cell apoptosis. This effect was closely correlated with the inhibition of NF- $\kappa \mathrm{B}$. Overall, these results suggest that $\mathrm{HO}-1 / \mathrm{CO}$ has potential clinical applications for use in the treatment of intestinal dysfunction.

\section{ACKNOWLEDGMENTS}

The present study was supported by grants from the National Natural Science Foundation of China (No. 81670479) and the Liaoning Provincial Research Program (No. 2016010223-301).

\section{DISCLOSURE}

The authors declare that they have no competing interests as defined by Molecular Medicine, or other interests that might be perceived to influence the results and discussion reported in this paper.

\section{REFERENCES}

1. Bona S, et al. (2012) Effect of antioxidant treatment on fibrogenesis in rats with carbon tetrachloride-induced cirrhosis. ISRN Gastroenterol. 2012:762920.

2. Ljubuncic P, Tanne Z, Bomzon A. (2000) Evidence of a systemic phenomenon for oxidative stress in cholestatic liver disease. Gut. 47:710-6.

3. Slocum M, Sittig K, Specian R, Deitch E. (1992) Absence of intestinal bile promotes bacterial translocation. Am. Surg. 58:305-10.

4. Ogata Y, et al. (2003) Role of bile in intestinal barrier function and its inhibitory effect on bacterial translocation in obstructive jaundice in rats. J. Surg. Res. 115:18-23.

5. Ridlon JM, Kang DJ, Hylemon PB, Bajaj JS. (2014) Bile acids and the gut microbiome. Curr. Opin. Gastroenterol. 30:332-8.

6. Schnabl B, Brenner DA. (2014) Interactions between the intestinal microbiome and liver diseases. Gastroenterology. 146:1513-24.

7. Szabo G. (2015) Gut-liver axis in alcoholic liver disease. Gastroenterology. 148:30-6.

8. ELamin EE, Masclee AA, Dekker J, Jonkers DM. (2013) Ethanol metabolism and its effects on the intestinal epithelial barrier. Nutr. Rev. 71:483-99.

9. Oshima T, Miwa H. (2016) Gastrointestinal mucosal barrier function and diseases. J. Gastroenterol. 51:768-78.

10. Chang M, Jing X, Sharma V, Habtezion A. (2015) Protective role of hemeoxygenase-1 in gastrointestinal diseases. Cell. Mol. Life Sci. 72:1161-73.
11. Bakhautdin B, et al. (2014) Protective role of HO-1 and carbon monoxide in ethanol-induced hepatocyte cell death and liver injury in mice. J. Hepatol. 61:1029-37.

12. Farombi EO, Surh YJ. (2006) Heme oxygenase-1 as a potential therapeutic target for hepatoprotection. J. Biochem. Mol. Biol. 39:479-91.

13. Yang H, et al. (2012) Heme oxygenase-1 prevents liver fibrosis in rats by regulating the expression of PPAR $\gamma$ and NF-кB. World J. Gastroenterol. 18:1680-88.

14. Malaguarnera L, Madeddu R, Palio E, Arena N, Malaguarnera M. (2005) Heme oxygenase-1 levels and oxidative stress-related parameters in non-alcoholic fatty liver disease patients. J. Hepatol. 42:585-91.

15. Zeng Z, Huang HF, Chen MQ, Song F, Zhang YJ (2010) Heme oxygenase-1 protects donor livers from ischemia/reperfusion injury: the role of Kupffer cells. World J. Gastroenterol. 16:1285-92.

16. Yeh CH, Yang JJ, Yang ML, Li YC, Kuan YH. (2014) Rutin decreases lipopolysaccharideinduced acute lung injury via inhibition of oxidative stress and the MAPK-NF-kappaB pathway. Free Radix. Biol. Med. 69:249-57.

17. Chi X, et al. (2015) Elevation of HO-1 expression mitigates intestinal ischemia-reperfusion injury and restores tight junction function in a rat liver transplantation model. Oxid. Med. Cell. Longeval. 2015:986075.

18. Wang F, Duan ZJ, Sun YJ. (2009) Influence of heme oxygenase-1 expression on immune liver fibrosis induced by cobalt protoporphyrin in rats. World J. Gastroenterol. 15:3009-14.

19. Wang QM, et al. (2013) Inhibiting heme oxygenase-1 attenuates rat liver fibrosis by removing iron accumulation. World J. Gastroenterol. 19:2921-34.

20. True AL, et al. (2007) Heme oxygenase-1 deficiency accelerates formation of arterial thrombosis through oxidative damage to the endothelium, which is rescued by inhaled carbon monoxide. Circ. Res. 101:893-901.

21. Motterlini R, Otterbein LE. (2010) The therapeutic potential of carbon monoxide. Nat. Rev. Drug. Discov. 9:728-43.

22. Babu D, Motterlini R, Lefebvre RA. (2015) CO and CO-releasing molecules (CO-RMs) in acute gastrointestinal inflammation. Br. J. Pharmacol. 172:1557-73.

23. Takagi T, Uchiyama K, Naito Y. (2015) The therapeutic potential of carbon monoxide for inflammatory bowel disease. Digestion. 91:13-8.

24. Mu X, et al. (2014) Protective effects of carbon monoxide-releasing molecule- 2 on the barrier function of intestinal epithelial cells. PLoS One. 9:e104032.

25. Zhang S, et al. (2015) Carbon monoxide-releasing molecule-2 reduces intestinal epithelial tight-junction damage and mortality in septic rats. PloS One. 10:e145988.

26. Wang QM, et al. (2013) Heme oxygenase/carbon monoxide pathway inhibition plays a role in ameliorating fibrosis following splenectomy. Int. J Mol. Med. 31:1186-94. 
27. Amersi F, et al. (1999) Upregulation of heme oxygenase- 1 protects genetically fat Zucker rat livers from ischemia/reperfusion injury. J. Clin. Invest. 104:1631-9

28. Chiu CJ, McArdle AH, Brown R, Scott HJ, Gurd FN. (1970) Intestinal mucosal lesion in low-flow states. I. A morphological, hemodynamic, and metabolic reappraisal. Arch. Surg. 101:478-83.

29. Wang P, et al. (2016) Promising therapy candidates for liver fibrosis. Front. Physiol. 7:47.

30. Cai SY, et al. (2017) Bile acids initiate cholestatic liver injury by triggering a hepatocyte-specific inflammatory response. JCI Insight. 2:e90780.

31. Kundu S, Kumar S, Bajaj A. (2015) Cross-talk between bile acids and gastrointestinal tract for progression and development of cancer and its therapeutic implications. IUBMB Life. 67:514-23.

32. Somi MH, et al. (2013) Lipoic acid prevents hepatic and intestinal damage induced by obstruction of the common bile duct in rats. Eur. Rev. Med. Pharmacol. Sci. 17:1305-10.

33. Chazouillères O. (2016) Novel aspects in the management of cholestatic liver diseases. Dig. Dis. 34:340-6.

34. Zahiri HR, Perrone EE, Strauch ED. (2011) Bile salt supplementation acts via the farnesoid $X$ receptor to alleviate lipopolysaccharide-induced intestinal injury. Surgery. 150:480-9.

35. Origassa CS, Camara NO. (2013) Cytoprotective role of heme oxygenase- 1 and heme degradation derived end products in liver injury. World. J. Hepatol. 5:541-9.

36. Barikbin R, et al. (2012) Induction of heme oxygenase 1 prevents progression of liver fibrosis in Mdr2 knockout mice. Hepatology. 55:553-62

37. Wang RQ, et al. (2011) Induction of heme oxygenase-1 protects against nutritional fibrosing steatohepatitis in mice. Lipids Health Dis. 10:31

38. Froh $\mathrm{M}$, et al. (2007) Heme oxygenase-1 overexpression increases liver injury after bile duct ligation in rats. World J. Gastroenterol. 13:3478-86

39. Mehta G, Mookerjee RP. (2014) Breaking badthe two sides of gut microbiota in portal hypertension. Liver Int. 34:1295-7.

40. Liu Z, Li N, Neu J. (2005) Tight junctions, leaky intestines, and pediatric diseases. Acta Paediatr. 94:386-93.

41. Su L, et al. (2009) Targeted epithelial tight junction dysfunction causes immune activation and contributes to development of experimental colitis. Gastroenterology. 136:551-63.

42. Xiao YT, Yan WH, Cao Y, Yan JK, Cai W. (2016) Neutralization of IL- 6 and TNF- $\alpha$ ameliorates intestinal permeability in DSS-induced colitis. Cytokine. 83:189-92.

43. He F, et al. (2012) Mechanisms of tumor necrosis factor-alpha-induced leaks in intestine epithelial barrier. Cytokine. 59:264-72.

44. Mir H, et al. (2016) Occludin deficiency promotes ethanol-induced disruption of colonic epithelial junctions, gut barrier dysfunction and liver damage in mice. Biochim. Biophys. Acta. 1860:765-74.
45. Smale ST. (2011) Hierarchies of NF-kappa B target-gene regulation. Nat. Immunol. 12:689-94.

46. Chi PL, et al. (2014) HO-1 induction by CORM2 attenuates TNF- $\alpha$-induced cytosolic phospholipase A2 expression via inhibition of PKC $\alpha$ dependent NADPH oxidase/ROS and NF-kB. Mediators Inflamm. 2014:279171.

47. Xiong J, et al. (2017) Luteolin protects mice from severe acute pancreatitis by exerting HO-1-mediated anti-inflammatory and antioxidant effects. Int. J. Mol. Med. 39:113-25.

48. Alvira CM. (2014) Nuclear factor-kappa-B signaling in lung development and disease: one pathway, numerous functions. Birth Defects Res. A Clin. Mol. Teratol. 100:202-16.

Cite this article as: Zhang L, et al. (2017) The protective effect of heme oxygenase-1 against intestinal barrier dysfunction in cholestatic liver injury is associated with NF-KB inhibition. Mol. Med. 23:215-24. 